\title{
Quantitative magnetic resonance imaging of the knee: a method of measuring response to intra-articular treatments
}

\author{
P Creamer, M Keen, F Zananiri, J C Waterton, R A Maciewicz, C Oliver, P Dieppe, I Watt
}

Department of Rheumatology, Bristol Royal Infirmary, Bristol

P Creamer

P Dieppe

\section{Department of} Medical Physics and Bioengineering, Bristol General Hospital, Bristol $M$ Keen

F Zananiri

Vascular,

Inflammatory and

Musculo-Skeletal

Research Department, Zeneca

Pharmaceuticals, Cheshire

J C Waterton

R A Maciewicz

Department of Radiodiagnosis, Bristol Royal Infirmary, Bristol C Oliver

I Watt

Correspondence to: Dr P Creamer, Division of Rheumatology, University of Maryland, Rm 8-34 MSTF, 10 South Pine St, Baltimore MD 21201, USA.

Accepted for publication 18 March 1997

\begin{abstract}
Objectives-To investigate the potential of quantitative magnetic resonance imaging (MRI) to differentiate between therapeutically induced changes in inflammation and synovial proliferation in rheumatoid arthritis (RA) of the knee.

Methods-MRI of the knee was performed on patients with RA before and one week after injection with corticosteroid (triamcinolone acetonide, TA group, $n=9$ ) and before, four, and 12 weeks after injection with yttrium-90 plus TA (TA+Y group, $\mathbf{n}=7$ ). MRI scans were analysed by subjective visual grading by a trained observer and by computer aided quantitation for three features: synovial fluid volume, synovial pannus volume, and synovial enhancement after intravenous contrast agent.
\end{abstract}

Results-All TA subjects improved clinically at one week but the effects of TA+Y were more variable. TA significantly reduced synovial enhancement and effusion volume, whereas $T A+Y$ at 12 weeks tended to increase synovial enhancement and decrease pannus volume. Quantitative MRI values agreed well with subjective assessment of scans. Comparison of calculated change on MRI scan before and immediately after aspiration with actual volume aspirated showed high correlation $(r=0.96)$.

Conclusions-Quantitative MRI correlates with subjective visual assessment and, at least for synovial fluid, is accurate. MRI can differentiate actions of two therapeutic modalities on various pathological processes and is sensitive enough to detect change after one week. With the additional advantage of lack of observer bias, it will probably become a useful tool in the development and assessment of existing and novel treatments.

(Ann Rheum Dis 1997;56:378-381)

The pathology of knee joint arthritis reflects a number of different but interrelated processes including inflammation, synovial proliferation, vascular changes, and destruction of cartilage and bone. Therapeutic interventions are likely to act at specific points. Current methodologies such as clinical measures, measurement of the acute phase response, and plain radiography fail to differentiate between inflammation and synovial proliferation.

Magnetic resonance imaging (MRI) is unique in its ability to visualise bone, cartilage, synovium, and fluid, particularly when intravenous contrast (gadopentetate dimeglumine, GdDTPA) is used. ${ }^{12}$ There is now good evidence that GdDTPA enhancement reflects areas of synovial inflammation as confirmed by biopsy and histology. ${ }^{2-4}$ Newer sequences have also improved the discriminatory ability of MRI. $^{25}$

The mode of action of intra-articular corticosteroid in RA is uncertain. A reduction in blood flow can be demonstrated by scintigraphy, ${ }^{6}$ suggesting a rapid antiinflammatory effect. The radioisotope yttrium-90 $\left({ }^{90} \mathrm{Y}\right)$, used to treat persistently inflamed joints since 1963, seems to produce effects at three months, lasting for up to a year. ${ }^{7}$ The duration of such an effect suggests an action on proliferating cells and pannus formation.

The aims of this study were to compare subjective assesment of MRI features with quantitative assessment and to investigate the potential of quantitative MRI to differentiate between therapeutically induced changes in inflammation (using corticosteroid injection as a model) and synovial proliferation (using ${ }^{90} \mathrm{Y}$ ) in the knee joint of patients with rheumatoid arthritis (RA).

\section{Methods}

PATIENTS

Subjects $(n=16)$ were consecutive patients with $\mathrm{RA}$ in whom either corticosteroid or ${ }^{90} \mathrm{Y}$ injection to a single knee had been recommended by their physician. Nine were assessed before and one week after aspiration and injection with $40 \mathrm{mg}$ triamcinolone acetonide (TA group). The remaining patients $(n=7)$ were assessed before, four, and 12 weeks after injection with ${ }^{90} \mathrm{Y}$ (200 MBq per joint) plus triamcinolone acetonide $40 \mathrm{mg}$ (TA+Y group). In all cases the maximum amount of fluid that could be aspirated was removed from the joint. The timing of assessments was chosen to represent the anticipated peak action of the treatments.

The mean age of subjects in the TA group was 59 years (range 27-74) with a disease duration of 9.8 years (range $2-28$ ). The TA+Y group were older at 66 years (range 38-81) with disease duration of 25.6 years (range 
Table 1 Comparison of subjective grading of MRI features and quantitated values

\begin{tabular}{llllll}
\hline MRI parameter & Subjective grade & $\begin{array}{l}\text { Number of } \\
\text { observations }\end{array}$ & Median & Range & p Value (Kruskal -Wallis) \\
\hline Effusion volume & 0 & 10 & $2.25 \mathrm{ml}$ & $0.6-8.0$ & \\
& 1 & 13 & $9.2 \mathrm{ml}$ & $0.2-22.7$ & \\
Pannus volume & 2 & 9 & $28.9 \mathrm{ml}$ & $11.4-42.7$ & $\mathrm{p}<0.001$ \\
& 3 & 6 & $56.6 \mathrm{ml}$ & $37.9-159.4$ & \\
Enhancement & 1 & 6 & $75.5 \mathrm{ml}$ & $57-104$ & \\
& 2 & 10 & $149.0 \mathrm{ml}$ & $81-189$ & $\mathrm{p}=0.0016$ \\
& 3 & 11 & $135.0 \mathrm{ml}$ & $30-212$ & \\
& 1 & 7 & $186.0 \mathrm{ml}$ & $112-276$ & $8-86$ \\
\end{tabular}

7-47). Informed written consent and ethics committee approval was obtained.

Clinical scoring, before each scan, comprised patient's assessment of rest, use and night pain, early morning stiffness, and inactivity stiffness in the index knee. Each symptom was graded $0-3$ resulting in a total maximum pain/stiffness score of 15 . Soft tissue swelling was recorded $(0,1$ or 2$)$, as was patient's overall assessment of change (much better, better, same, worse, much worse, scored 1-5 respectively).

MAGNETIC RESONANCE IMAGING

A Siemens Impact $1.0 \mathrm{~T}$ scanner was used. Sequences, chosen for their ability to visualise synovium and effusions, comprised $\mathrm{T} 1$ weighted ( $\mathrm{TR}=400, \mathrm{TE}=15, \mathrm{FOV}$ (field of view) 250) and $\mathrm{T} 2$ weighted $(\mathrm{TR}=1800$, $\mathrm{TE}=90$, FOV 250). PSIF 3D gradient echo ( $\mathrm{TR}=17, \mathrm{TE}=7$, flip angle 30, FOV 250) scans were performed before and immediately after aspiration of effusions. Post contrast (GdDTPA, $0.1 \mathrm{mmol} / \mathrm{kg}$ ) dynamic acquisition sequences (FLASH 3D: TR $=30, \mathrm{TE}=6$, flip angle 30, FOV 250) were performed to allow rate of enhancement to be calculated: 20 measurements, each taking 14 seconds to acquire, were made with 0.3 seconds between each set. The T1 weighted sequence was also repeated after enhancement. Four $\mathrm{mm}$ sagittal slices were taken throughout with the exception of PSIF 3D and FLASH 3D for which $2 \mathrm{~mm}$ slices were taken.

Two methods of measuring synovial pannus volume, fluid volume, and enhancement were compared: visual assessment by a radiologist and computer aided quantitation. The following features were graded subjectively: effusion (0-3), pannus (0-3) and degree of enhancement $(0-2)$. Quantitation of fluid and pannus volumes used methods similar to those published elsewhere. ${ }^{289}$ Briefly, fluid volume was obtained from T2 weighted (PSIF) scans by manual segmentation using Siemens image analysis software. The area calculated for each slice was multiplied by the slice separation to give the total volume. Pannus volume was calculated after completion of the dynamic acquisition sequences (about five minutes after Gd DTPA injection) from T1 weighted sequences, using an image processing station ('Mipron', Kontron). In each slice, thresholds were applied to exclude noise and fat and a circular region of interest (ROI) to include the joint space was imposed. For pixels within this ROI and between these thresholds, a parametric image was calculated via: ((after before)/before) $) \times 100 \%$. Pixels enhancing by more than $50 \%$ and less than $300 \%$ were counted. Again, the area of pannus was outlined and calculated for each slice and finally multiplied by the slice thickness. Quantitation of enhancement used signal intensity plots from a ROI in the suprapatellar pouch (the most homogeneous region in terms of enhancement). Two measurements were made: the percentage increase in signal intensity (SI) and the maximum gradient of increase in signal intensity, measured in units/second. All quantitative analysis was performed blind to timing and nature of treatment.

REPRODUCIBILITY

Intraobserver reliability for subjective analysis was assessed before the study by grading 10 scans for the three features on two occasions: of 30 replicates, a different grade were assigned on four occasions, in all cases by a difference of just one grade. Replicate studies of pannus volume calculation (four subjects repeated five times) gave a coefficient of variation of $0.89 \%$; for synovial fluid volume (single subject, eight replicates) CV was $4.5 \%$

STATISTICAL ANALYSIS

To compare aspirated and calculated fluid volumes the approach of Altman and Bland ${ }^{10}$ was used after first talking the log of volume plus 1 . The relation between subjective grades and quantitative values was examined by the Kruskal-Wallis test. This test assumes independence of observations. Although up to three observations are included from the same

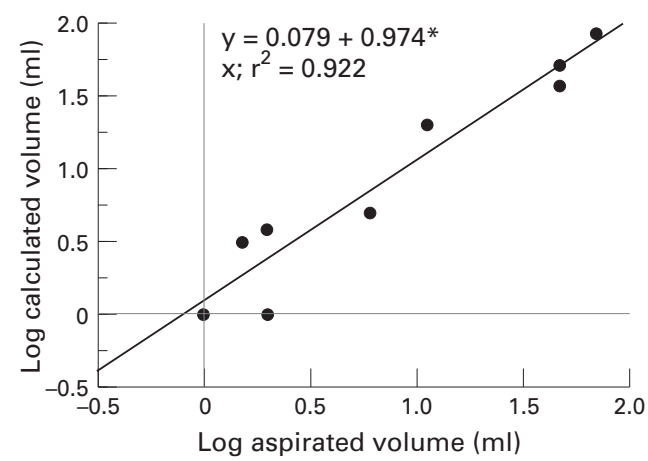

Figure 1 Comparison of aspirated volume of synovial fluid with calculated change in volume on MRI. Log transformations used. $r=0.9604$. 


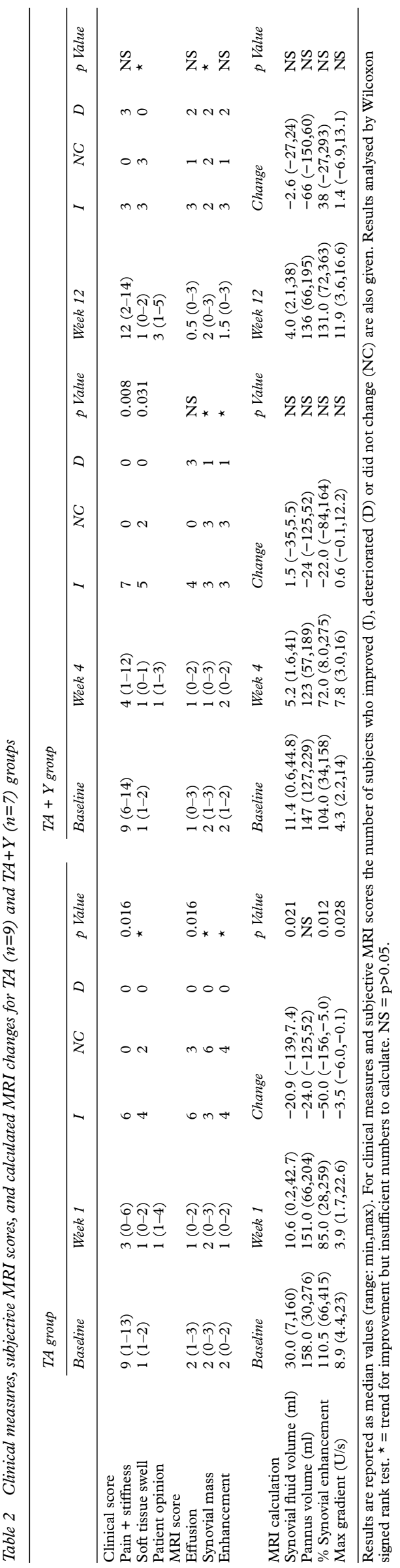

individual these are separated by up to three months. Limiting analysis to one observation per subject gave similar results. The clinical scores and quantitative MRI data were analysed using the Wilcoxon signed rank rest on the change from baseline.

\section{Results}

Table 1 shows the relation between subjective scores and calculated values. In addition, there was a strong relation between the degree of soft tissue swelling and calculated effusion volume $(\mathrm{p}=0.0004)$ and pannus volume $(\mathrm{p}=0.021)$, but not enhancement $(\mathrm{p}=0.23)$.

External validation was sought for fluid volume by comparing the volume of fluid aspirated with the calculated change in volume determined by MRI taken before and after aspiration (fig 1). Log regression plot gave a correlation of 0.96 . There was no statistical evidence of differential or overall bias.

In the TA study, all patients improved by pain and stiffness score and most felt 'much better' one week after corticosteroid injection (table 2). Soft tissue swelling decreased or did not change in all subjects. MRI showed a significant reduction in synovial fluid volume both by score and quantitation (median fall $20.9 \mathrm{ml}$ ). Synovial enhancement also fell in all subjects as measured by both percentage enhancement (median fall 50.0\%) and by maximum gradient (median 3.5 units/s). Synovial pannus was not affected significantly by corticosteroid treatment. The measured volume actually rose in two subjects.

In the TA + Y study, pain and stiffness, soft tissue swelling, and patient's opinion also improved at four weeks. However, there were no significant changes in MRI either by score or quantitative values. At 12 weeks, the improvement in patients' pain and stiffness had been lost. Only two of the six patients felt better. Again, at 12 weeks MRI values were not significantly different from baseline. Enhancement actually increased while pannus volume decreased (both non-significant). The two patients who reported feeling better had the largest calculated fall in pannus at 12 weeks.

\section{Discussion}

Our results suggest that the volume of synovial fluid and proliferative synovial tissue and the degree of enhancement after GdDTPA injection can be reliably quantitated using MRI. We have shown good correlation between calculated and actual volume of fluid removed, providing some external validity. There is also a good relation between subjective assessment of features and calculated values. Finally, biological plausibility is suggested by the finding that soft tissue swelling relates closely to calculated volumes for synovial fluid and, to a lesser extent, pannus but not to enhancement.

There have been several previous studies in which MRI has been used to evaluate drug therapy. ${ }^{112}$ Corticosteroids have been shown to reduce GdDTPA enhancement ${ }^{13}$ and pannus volume in the knee. ${ }^{14}$ Osmic acid 
synovectomy was shown to reduce pannus volume without affecting enhancement. ${ }^{15}$

Quantitative MRI is technically difficult and makes certain assumptions. For example, Gd-DTPA may diffuse into fluid resulting in peripheral enhancement of effusion and part of the synovial fluid being measured as pannus. Diffusion is increased with mobilisation of the knee and in the presence of synovitis. In our study, scans were obtained shortly after injection and patients remained resting. Other potential difficulties include reproducibility of image slices taken at different times and definition of tissue to be analysed. In this study, pannus and effusion measurements were made for a volume including the entire knee, thus eliminating errors arising from slice irreproducibility.

Improvement was expected in patients treated with corticosteroids and, indeed, was seen in all clinical parameters. These were reflected in MRI changes, which also allowed differential effects to be studied. The corticosteroid clearly reduced fluid volume and gadolinium enhancement. This observed reduction may have resulted from corticosteroid, aspiration of fluid or a combination of both. However, as synovial fluid has a rapid turnover, re-accumulation would result unless the drug was exerting a therapeutic effect. Both the gradient of enhancement, (which reflects perfusion) and the maximum enhancement (reflecting both perfusion and diffusion) were reduced supporting a role for corticosteroids in reducing inflammation. The effect of TA on pannus was not significant. The response in the $\mathrm{TA}+\mathrm{Y}$ group at four weeks may reflect persistent effects of corticosteroid given simultaneously with ${ }^{90} \mathrm{Y}$, or even a placebo response. This improvement was not maintained at 12 weeks nor was it, in general, matched by MRI changes. The two subjects who reported feeling better at 12 weeks had the greatest reduction in pannus, as previously reported in osmic acid synovectomy. ${ }^{15}$ It is possible that the timing of our assessments missed the peak action of the drug. Our study, however, had very small numbers and may simply have lacked the power to detect an effect of ${ }^{90} \mathrm{Y}$.

Quantitative MRI has advantages of objectivity and lack of observer bias. It is capable of demonstrating changes in synovial pathology over a short time period and can differentiate between effects of intra-articular treatment on inflammation and pannus forma- tion. MRI features seem to correlate with clinical improvement. While the end result of pain felt by the patient remains an important outcome measure, the ability to detect changes at the site of pathology allows a more discriminating approach to development and assessment of treatments.

We are grateful to and acknowledge the contribution of: Brian Middleton for statistical advice, Sally Speake for help with MRI reporting, Janet Cushnaghan for clinical assessments of reporting, Janet Cushnaghan for clinical assessments of patients, Liz Berry for initial work on MRI processing and
Diane Pressdee, Debbie Macey and Kath Roberts of the Department of Clinical Radiology, Bristol Royal Infirmary. This Department of Clinical Radiology, Bristol Royal Infirmary. This work was funded in part by
Park, Cheshire SK10 4TG.

1 Adam G, Dammer M, Bohndorf K, Christoph R, Fenke F, Gunther RW. Rheumatoid arthritis of the knee: value of gadopentetate dimeglumine enhanced MR imaging. A J R Am J Roenterol 1991;156:125-9.

2 Konig H, Sieper J, Wolf K-J. Rheumatoid arthritis: evaluation of hypervascular and fibrous pannus with dynamic MR imaging enhanced with Gd-DTPA. Radiology 1990;176:473-7.

3 Gaffney K, Cookson J, Blake D, Coumbe A, Blades S. Quantification of rheumatoid synovitis by magnetic resonance imaging. Arthritis Rheum 1995;38:1610-7.

4 Tamai K, Yamato M, Yamaguchi T, Ohno W. Dynamic magnetic resonance imaging for the evaluation of synovitis in patients with rheumatoid arthritis. Arthritis Rheum in patients with

5 Peterfy CG, Majumdar S, Lang P, van Dijke CF, Sack K, Genant HK. MR imaging of the arthritic knee: improved discrimination of cartilage, synovium and effusion with pulsed saturation transfer and fat suppressed T1- weighted sequences. Radiology 1994;191:413-9.

6 McCrae FC, Palmer M, Shouls JC, Watt I, Dieppe P. Scintigraphic assessment of synovial and bone responses after intra-articular yttrium90 therapy. (Abstract). Br J Rheumatol 1988;27 (suppl 1):23.

7 Will R, Laing B, Edelman J, Lovegrove F, Surveyor I. Comparison of two yttrium-90 regimens in inflammatory and osteoarthropathies. Ann Rheum Dis 1992;51:262-5.

8 Heuck AF, Steiger P, Stoller DW, Gluer CC, Genant HK. Quantification of knee joint fluid volume by MR imaging and CT using three-dimensional data processing. J Comput Assist Tomogr 1989;13:287-93.

9 Waterton JC, Rajanyagam V, Ross BD, Brown D, Whittemore A, Johnstone D. Magnetic resonance methods for more A, Johnstone D. Magnetic resonance methods for measuring disease progression in rh

10 Altman DG, Bland JM. Measurement in medicine: the analysis of method comparison studies. The Statistician 1983;32:307-17.

11 Beltran J, Caudill JL, Herman LA, Kantor SM, Hudson PN, Noto AM, et al. Rheumatoid arthritis: MR imaging manifestations. Radiology 1987;165:153-7.

12 Paul PK, Stevens RM, Mezrich RS, Goldberg RL, Doughty JR, Dunton AW, et al. Magnetic resonance imaging evaluation of therapy in RA. Clin Pharmacol Ther 1992; 51:193.

13 Ostergaard M, Stoltenberg M, Henriksen O, Lorenzen I. Quantitative assessment of synovial inflammation by dynamic gadolinium-enhanced magnetic resonance imaging. A study of the effect of intra-articular methylpreding. A study of the effect of intra-articular methylpred-
nisolone on the rate of early synovial enhancement. Br J

14 Kursunogulu-Brahme S, Riccio T, Weisman MH, Resnick D, Zvaifler N, Sanders ME, et al. Rheumatoid knee: role of gadolinium enhanced MR imaging. Radiology 1990; 176:831-5.

15 Ostergaard M, Stoltenberg M, Gideon P, Wieslander S, Sonne-Holm S, Kryger P, et al. Effect of intraarticular osmic acid on synovial membrane volume and inflammation, determined by magnetic resonance imaging. Scand J Rheumatol 1995;24:5-12. 